\title{
Prediction Model for the Risk of Scapular Winging in Young Women Based on the Decision Tree
}

\author{
Gyeong-tae Gwak ${ }^{1,2}$, BPT, PT, Sun-hee Ahn ${ }^{1,2}$, MSc, PT, Jun-hee Kim ${ }^{1,2}$, BPT, PT, Young-soo Weon ${ }^{1,2}$, BHSc, PT, \\ Oh-yun Kwon ${ }^{1,3,4}$, PhD, PT
}

${ }^{1}$ Kinetic Ergocise Based on Movement Analysis Laboratory, ${ }^{2}$ Department of Physical Therapy, The Graduate School, Yonsei University, ${ }^{3}$ Department of Physical Therapy, College of Health Science, Yonsei University, ${ }^{4}$ Department of Ergonomic Therapy, The Graduate School of Health and Environment, Yonsei University, Wonju, Korea

\author{
Article Info \\ Received January 16, 2020 \\ Revised February 13, 2020 \\ Accepted March 4, 2020 \\ Corresponding Author \\ Oh-yun Kwon \\ E-mail: kwonoy@yonsei.ac.kr \\ https://orcid.org/0000-0002-9699-768X
}

\section{Key Words}

Decision tree

Musculoskeletal disease

Physical examination
Background: Scapular winging (SW) could be caused by tightness or weakness of the periscapular muscles. Although data mining techniques are useful in classifying or predicting risk of musculoskeletal disorder, predictive models for risk of musculoskeletal disorder using the results of clinical test or quantitative data are scarce.

Objects: This study aimed to (1) investigate the difference between young women with and without SW, (2) establish a predictive model for presence of SW, and (3) determine the cutoff value of each variable for predicting the risk of SW using the decision tree method.

Methods: Fifty young female subjects participated in this study. To classify the presence of SW as the outcome variable, scapular protractor strength, elbow flexor strength, shoulder internal rotation, and whether the scapula is in the dominant or nondominant side were determined.

Results: The classification tree selected scapular protractor strength, shoulder internal rotation range of motion, and whether the scapula is in the dominant or nondominant side as predictor variables. The classification tree model correctly classified $78.79 \%(p=0.02)$ of the training data set. The accuracy obtained by the classification tree on the test data set was $82.35 \%(p=0.04)$.

Conclusion: The classification tree showed acceptable accuracy $(82.35 \%)$ and high specificity $(95.65 \%)$ but low sensitivity (54.55\%). Based on the predictive model in this study, we suggested that $20 \%$ of body weight in scapular protractor strength is a meaningful cutoff value for presence of SW.

\section{INTRODUCTION}

Scapular winging (SW) is an abnormal scapulothoracic posture that refers to prominence of the medial border or inferior angle of the scapula from thoracic wall [1]. Abnormal scapular position and movement are associated with scapular dysfunction and imbalance of the periscapular muscles, resulting in shoulder pain and impingement syndrome [2,3]. Although multiple factors may cause SW, it can result from weakness of the scapular stabilizers without bone, joint, and neurological factors $[1,2]$.

Soft tissue mechanisms for SW involve tightness or weakness of periscapular muscles [4]. The serratus anterior muscle is considered a primary scapular stabilizer during arm movement, which stabilizes the scapula against the thoracic wall [5]. Weakness of the serratus anterior muscle could lead to insufficient scapular posterior tilt and upward rotation during arm movements, causing dyskinesis [6,7]. In addition, weakness of scapular stabilizers including serratus anterior and lower trapezius may lead to pseudoweakness of the upper extremity muscles because of lack of proximal stabilization to provide a stable origin [8,9]. Inflexibility and stiffness of the pectoralis minor and biceps short head could result in scapular anterior tilt and protraction due to their pull on the coracoid process $[4,10]$. Moreover, glenohumeral internal rotation deficit due to posterior shoulder tightness could create compensatory scapu- 
lar anterior tilts [11]. The scapula moves with the humerus as the arm is internally rotated, which creates a "windup" of the scapula on the thorax with reduced humeral internal rotation and horizontal abduction [4,11].

Repetitive stress has been considered as an important factor for musculoskeletal disorders [12]. Previous studies have noted that the dominant extremity often exhibits accentuated dyskinetic findings attributed to overuse, muscle asymmetry, and differences in range of motion [13-15]. The loss of shoulder internal rotation range of motion (ROM) in dominant shoulder has been studied extensively in unilateral overhead athletes such as baseball players and tennis players [13,16,17]. The results of the studies reported that dominant shoulder had significantly lower shoulder internal rotation ROM in unilateral overhead athletes. Also, Cieminski et al. [14] reported that the dominant shoulder had significantly less shoulder internal rotation ROM compared with the nondominant shoulder in nonathletic persons. Because the unwanted stress caused by the compensatory movements may vary depending on the degree of use of the arm [18], whether dominant or nondominant side could affect the prevalence of upper extremity musculoskeletal disorders [19].

Previous studies have proposed various methods in assessing SW, such as visual observations and 3D motion analysis systems. Visual observations are easy and inexpensive to implement but relatively subjective. In contrast, 3D motion analysis systems could provide quantitative data; however, such systems are impractical in clinical settings. Other assessment tools have been designed to measure the posterior displacement of the inferior angle of the scapula with reference to the posterior thoracic cage [20-23]. Weon et al. [23] designed a scapulometer to measure the posterior displacement of the scapular inferior angle relative to the thoracic wall. They also suggested that a posterior displacement $>2 \mathrm{~cm}$ indicates presence of SW. De Oliveira et al. [21] suggested that a $1.5 \mathrm{~cm}$ cutoff showed better sensitivity, specificity, and diagnostic performance than a 2 $\mathrm{cm}$ cutoff. Although the results of these tests could provide information on the presence of SW, additional tests were needed to examine the shoulder girdle function of patients.

The decision tree is one of the most popular classification techniques [24]. One of the main reasons is the ability of decision trees to present the results in a simple format, which is easy to interpret for experts [25]. In the decision tree model, for categorical variables, a branch is created for each known category, while, for continuous variables, the best split point is determined as the cutoff point for these variables [26]. Previous studies reported that data mining techniques are useful in classifying or predicting risk of musculoskeletal disorder [25,27-29]. However, most studies used questionnaire data or environmental variables $[25,27]$. The use of data mining techniques in classifying and predicting risk of musculoskeletal disorder using the results of clinical test or quantitative data is scarce [30]. Therefore, this study aimed to establish a predictive model for presence of SW, and determine the cutoff value of each variable for predicting the risk of SW using the decision tree method.

\section{MATERIALS AND METHODS}

\section{Subjects}

Fifty female subjects (mean age, $27 \pm 4$ years; range, 21-35) participated in this study. Subjects were excluded if they had (1) pain in the shoulder, elbow, or wrist joint; (2) previous history of shoulder, elbow, or wrist surgery; (3) problems such as tendonitis, adhesive capsulitis, or neurological pathology including damage to the long thoracic nerve. All variables were measured in both sides of all subjects. The data of both sides were used in the analysis because whether the scapula is in the dominant or nondominant side was one of predictor variables. Before the study, all subjects received explanations on all procedures of the study and signed an informed consent form approved by the Institutional Review Board of Yonsei University Mirae Institutional Review Board (approval No. 1041849201912-BM-177-01).

In measuring SW in the static condition, each subject stood in a neutral shoulder position with the elbow joint in $90^{\circ}$ flexion and the forearm in neutral position; then, a cuff weighing $5 \%$ of the body weight of each subject was placed on the distal wrist. Then, we measured the scapular posterior displacement (distance between the thoracic wall and inferior angle of the scapula) in all subjects using a modified scapulometer [31]. Subjects were assigned to the SW group (scapular posterior displacement $\geq 1.5 \mathrm{~cm}$ ) and without $\mathrm{SW}$ group (scapular posterior displacement $<1.5 \mathrm{~cm}$ ) based on the previous study conducted by de Oliveira et al. [21].

\section{Instruments}

The Smart KEMA strength sensor (KOREATECH, Inc., Seoul, 
Korea) was used to measure the maximal isometric scapular protractor and elbow flexor strength [32,33]. Data measured using the sensor were transferred to an Android tablet PC (Galaxy Tab A6 10.1; Samsung, Inc., Seoul, Korea) via Bluetooth network and analyzed using the Smart KEMA application (KOREATECH, Inc.). Data were recorded for 5 seconds, and the mean value of the middle 3 seconds was recorded.

The Smart KEMA motion sensor (KOREATECH, Inc.) was used to measure shoulder internal rotation ROM. The motion sensor contained a tri-axillar gyroscope, magnetometer, and accelerometer and signal converter and signal transmission sensor. Data from the motion sensor were recorded at a $25 \mathrm{~Hz}$ sampling frequency and transmitted to an Android tablet PC with the Smart KEMA software [34].

\section{Procedures}

Subjects were placed in supine position on a table with $90^{\circ}$ shoulder flexion and full elbow extension to measure maximal isometric scapular protractor strength (Figure 1). In measuring isometric strength during maximal scapular protraction, the subject grasped a handle connected to the Smart KEMA strength sensor, and then the arm length was adjusted. The Smart KEMA strength sensor was attached to a grounded vacuum lifter using a belt to provide a fixed point during measurements (Figure 1). To adjust the initial tension before strength measurements, the strength sensor maintained a tension of $2 \mathrm{kgf}$ when the handle was first grasped [32]. The maximal isometric scapular protractor strength was measured twice in

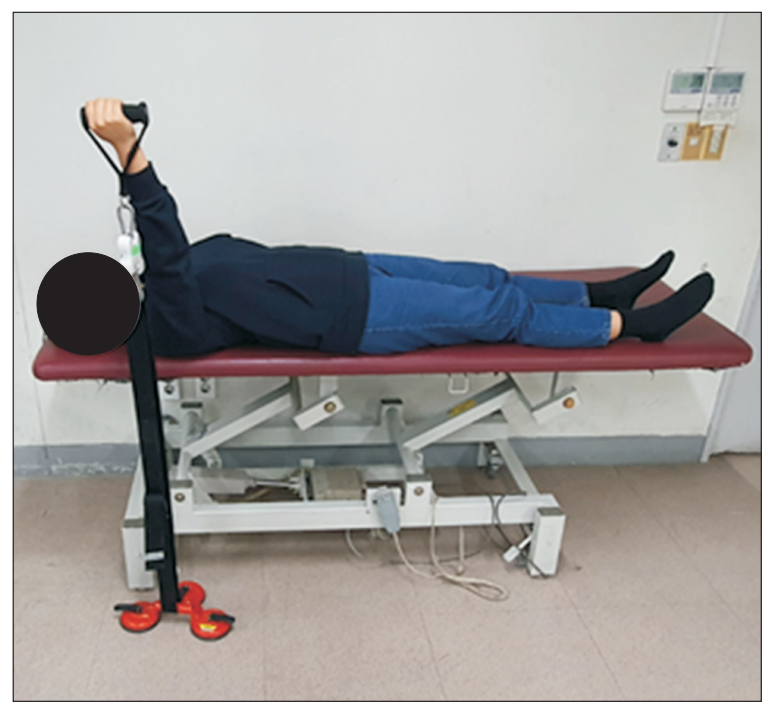

Figure 1. Maximal isometric scapular protractor strength measurement. each position, with a 3 minute rest period between measurements to prevent muscle fatigue. In each trial, the elbows were extended during exertion of maximal force against the grip handle in the forward direction.

Subjects were seated with the shoulder in the anatomical neutral position, elbow flexed to $90^{\circ}$, and forearm supinated to measure maximal isometric elbow flexor strength (Figure 2). To measure maximal isometric elbow flexor strength, a cuff connected to the Smart KEMA strength sensor was attached to the distal forearm of subjects. The Smart KEMA strength sensor was attached to a grounded vacuum lifter using a belt to provide a fixed point during measurements (Figure 2). To adjust initial tension before strength measurements, the strength sensor maintained a tension of $2 \mathrm{kgf}$ with elbow $90^{\circ}$ flexion [32]. Subjects performed isometric elbow flexion maximally against the fixed point. The humerus of test side was stabilized to maintain neutral shoulder position using opposite side hand during the measurement. The maximal isometric elbow flexor strength was measured twice in each position, with a 3-minute rest period between measurements to prevent muscle fatigue.

Shoulder internal rotation ROM was measured with the subject lying on the measurement side. The shoulder was flexed to $90^{\circ}$ with $0^{\circ}$ rotation, and the elbow was flexed to $90^{\circ}$ (Figure 3 ). The Smart KEMA motion sensor was attached to the wrist of subjects with a cuff. The examiner stabilized the head of the humerus to prevent anterior gliding and then passively internally rotated the humerus while maintaining $90^{\circ}$ shoulder and elbow flexion (Figure 3). Determination of shoulder internal

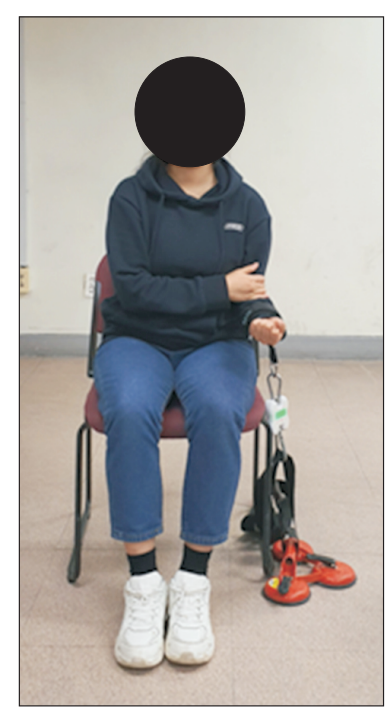

Figure 2. Maximal isometric elbow flexor strength measurement. 


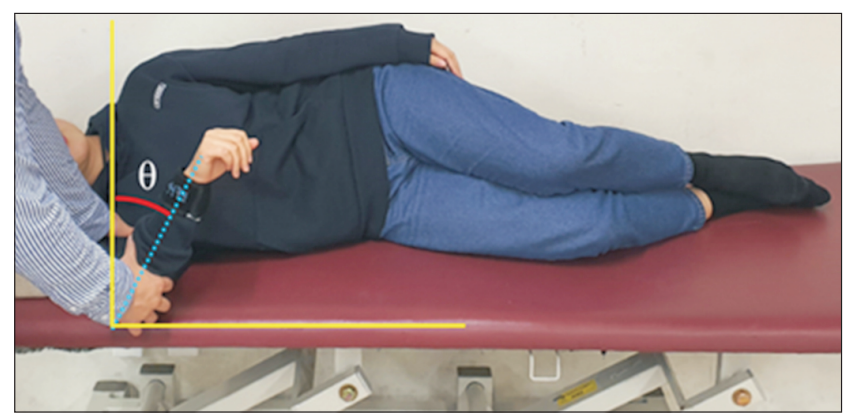

Figure 3. Shoulder internal rotation range of motion measurement.

rotation ROM based on the point of maximal internal rotation, where a distinct, firm end feel was noted by the examiner. When the maximal passive shoulder internal rotation was achieved, the data were collected and transmitted to the tablet.

\section{Statistical Analysis}

SPSS version 25.0 (IBM Co., Armonk, NY, USA) was used for the statistical analysis. The intraclass correlation coefficient (ICC 3, 1) model was used to test intra-rater reliability of measurements. Data normality was examined using the Kolmogorov-Smirnov test. Before establishing the prediction model, independent t-test was used to identify statistically significant differences in scapular protractor strength, elbow flexor strength, and shoulder internal rotation ROM between the two groups. The level of statistical significance was set at 0.05 .

A classification tree for presence of SW as outcome variable was derived using the rpart function of RStudio (version 1.1.463; RStudio, Inc., Boston, MA, USA) [35]. A classification tree selects the predictor variables that maximizes the decrease in impurity (minimization of the Gini index for categorical variables and the expected sum variances for continuous variables as node impurity criterion) [36]. In the classification with the presence of SW as outcome variable, scapular protractor strength, elbow flexor strength, shoulder internal rotation ROM, and whether the scapula is in the dominant or nondominant side were used as predictor variables. The strength values were divided by body weight of subjects for normalization. The subjects were randomly divided into a training data set (twothirds of the subjects) and test data set (the remaining third), each group with the same proportion in both data sets. Decision trees also have excellent prediction capabilities but have been criticized for overfitting and poor performance on ex-
Table 1. Characteristics of study subjects

\begin{tabular}{lccl}
\hline \multicolumn{1}{c}{ Variables } & With SW $(\mathrm{n}=33)$ & Without SW $(\mathrm{n}=67)$ & $\mathrm{p}$-value \\
\hline Age $(\mathrm{y})$ & $28.09 \pm 4.79$ & $26.64 \pm 3.94$ & $>0.05$ \\
Height $(\mathrm{cm})$ & $163.64 \pm 4.46$ & $162.51 \pm 4.12$ & $>0.05$ \\
Weight $(\mathrm{kg})$ & $60.27 \pm 9.96$ & $57.82 \pm 8.27$ & $>0.05$ \\
SPD $(\mathrm{cm})$ & $1.71 \pm 0.21$ & $0.85 \pm 0.42$ & $<0.05^{*}$ \\
\hline
\end{tabular}

Values are presented as mean \pm standard deviation. SW, scapular winging; SPD, scapular posterior displacement. *Represents significant differences.

Table 2. Comparison of scapular protractor strength, elbow flexor strength, and shoulder internal rotation range of motion between the two groups

\begin{tabular}{cccc}
\hline Variables & $\begin{array}{c}\text { With SW } \\
(\mathrm{n}=33)\end{array}$ & $\begin{array}{c}\text { Without SW } \\
(\mathrm{n}=67)\end{array}$ & p-value \\
\hline $\begin{array}{c}\text { Scapular protractor } \\
\text { strength (kgf) }\end{array}$ & $10.30 \pm 3.11$ & $12.07 \pm 3.96$ & $0.026^{*}$ \\
$\begin{array}{c}\text { Elbow flexor strength } \\
(\mathrm{kgf})\end{array}$ & $7.63 \pm 2.64$ & $9.15 \pm 3.21$ & $0.020^{*}$ \\
$\begin{array}{c}\text { Shoulder internal } \\
\text { rotation ROM }\left({ }^{\circ}\right)\end{array}$ & $43.60 \pm 12.67$ & $49.13 \pm 15.70$ & 0.062 \\
\hline
\end{tabular}

Values are presented as mean \pm standard deviation. SW, scapular winging; ROM, range of motion. *Represents significant differences.

tremely small data sets [25]. Therefore, fivefold cross-validation was used to evaluate the performance of the classification tree model.

\section{RESULTS}

The general characteristics of subjects are presented in Table 1. Of 100 cases, 33 were assigned into the SW group and 67 cases were assigned into the without SW group (without SW). All variables were normally distributed $(p>0.05)$. The ICC $(3,1)$ of the modified scapulometer was 0.867 (95\% CI, 0.808-0.908). There were no significant differences in age, height, or body weight between the two groups. There were significant differences in scapular protractor strength $(p=0.026)$ and elbow flexor strength $(p=0.020)$. However, there was no significant difference in shoulder internal rotation ROM between the two groups ( $\mathrm{p}=0.062)$ (Table 2). The ICCs $(3,1)$ were 0.923 (95\% CI, 0.887-0.947) for measuring scapular protractor strength, 0.926 (95\% CI, 0.892-0.950) for measuring elbow flexor strength, and 0.995 (95\% CI, 0.993-0.997) for measuring shoulder internal rotation ROM.

Figure 4 shows the classification tree for presence of SW, which had 4 terminal nodes. The classification tree for presence of SW showed scapular protractor strength as the first 




$\mathrm{SW}$, scapular winging; $\mathrm{Cl}$, confidence interval.

predictor of SW. Each node label showed the major subgroup (with SW or without SW). Below the node label, there was the number showing how many the major subgroup was observed in the node. Among the training data set (66 cases), 44 cases were without SW and 22 cases were with SW (Figure 4). The cutoff point of scapular protractor strength for absence of SW was $\geq 20 \%$ of body weight. In the subgroup of subjects with scapular protractor strength greater than or equal to $20 \%$ of body weight (27 cases), 23 cases were without SW and 4 cases were with SW. On the contrary, in the subgroup with lower scapular protractor strength (39 cases), 21 cases were without SW and 18 cases were with SW. Shoulder internal rotation ROM was the second predictor. The cutoff point of shoulder internal rotation ROM for absence of $S W$ was $\geq 56^{\circ}$. Eleven cases had shoulder internal rotation ROM greater than $56^{\circ}$, and 9 of them were without SW. Twenty-eight cases had shoulder internal rotation ROM less than $56^{\circ}$, and 16 of them were with SW. The last predictor variable was whether the scapula is in the domi-
Table 4. Performance of the classification tree as calculated during the model testing

\begin{tabular}{lccc}
\hline \multirow{2}{*}{$\begin{array}{c}\text { Calculated evaluation measures } \\
\text { on the test data set }\end{array}$} & \multicolumn{2}{c}{ Reference } \\
\cline { 3 - 4 } & Without SW & With SW \\
\hline Prediction (n) & Without SW & 22 & 5 \\
& With SW & 1 & 6 \\
Accuracy & & $95.65 \%$ & $54.55 \%$ \\
Overall accuracy/95\% Cl & $82.35 \% / 65.47 \%-93.24 \%$ \\
\hline
\end{tabular}

SW, scapular winging; $\mathrm{Cl}$, confidence interval.

nant or nondominant side. The scapula of the dominant side showed higher risk compared to the scapula of the nondominant side. In the terminal node, among 12 nondominant cases, 4 cases were with SW. On the contrary, among 16 dominant cases, 12 cases were with SW. Details of the tree divisions, with the respective predictors' cutoff points and number of cases classified in each subgroup according to selected predictors, are presented in Figure 4.

No information rate (classified by chance) of each data set showed $67 \%$ of accuracy due to imbalanced class (33 cases with SW, 67 cases without SW). The classification tree model correctly classified $78.79 \%(p=0.02)$ of the training data set $(66$ cases). The predicted SW risk levels based on the classification tree were verified against the test data set (34 cases). The accuracy obtained by the classification tree on the test data set was $82.35 \%(p=0.04)$. The confusion matrix between the predicted and actual SW risk levels indicates good overall accuracy in both data sets (Tables 3, 4). 


\section{DISCUSSION}

The results of this study showed that subjects with SW have reduced scapular protractor strength. This result is consistent with previous studies [37,38]. Kim et al. [38] reported significantly lower scapular protractor strength in male subjects with SW $(137.28 \pm 46.29 \mathrm{~N})$ than in the control group $(202.84 \pm$ $38.24 \mathrm{~N})$. Moreover, Kim et al. [38] suggested that $130 \mathrm{~N}$ as cutoff for presence of SW may indicate meaningful weakness of scapular protractor strength. In this study, the classification tree showed scapular protractor strength as the first predictor of SW. The cutoff value was $20 \%$ of the body weight. In the study conducted by Kim et al. [38], the mean body weight of SW group was $66.77 \pm 10.45 \mathrm{~kg}$. Thus, dividing $130 \mathrm{~N}$ by the mean body weight yields approximately $20 \%$ of body weight in male subjects, which is consistent with this study. Because the serratus anterior muscle is a scapular protractor and one of important scapular stabilizers, it would be reasonable that scapular protractor strength was selected as the first predictor.

The classification tree for presence of SW showed shoulder internal rotation ROM as the second predictor of SW. Previous studies reported that a large range of shoulder internal rotation ROM was required during activities of daily living. Magermans et al. [39] reported that $71^{\circ}$ shoulder internal rotation was observed during perineal care. Namdari et al. [40] revealed that $102^{\circ} \pm 7.7^{\circ}$ shoulder internal rotation (glenohumeral plus scapulothoracic) was required during 10 functional tasks including unhooking the bra. Because the scapula moves with the humerus as the arm is internally rotated more easily with reduced shoulder internal rotation, shoulder internal rotation deficit could lead to altered scapular kinematics $[4,11]$. Borich et al. [11] reported that there was a significant increase in scapular anterior tilt at end range shoulder internal rotation with the arm in $90^{\circ}$ of flexion or abduction in subjects with shoulder internal rotation deficit compared to controls. Therefore, shoulder internal rotation $\mathrm{ROM}<56^{\circ}$ might result in compensatory anterior tilts during functional activities of daily living, which could displace the scapular inferior angle away from the thoracic wall. Furthermore, considering that the last predictor variable selected by the classification tree is whether the scapula is in the dominant or nondominant side, repeated stress caused by shoulder internal rotation deficit seems to be important during functional activities of daily living. Although there was no significant difference in the result of independent t-test ( $p=0.062$ ) between the two groups, it would be reasonable that shoulder internal rotation ROM was the second predictor variable.

There was a significant difference in elbow flexor strength between the two groups. However, elbow flexor strength was not included as a predictor variable. Weakness of scapular stabilizer may lead to pseudo weakness of the upper extremity muscles because of lack of proximal stabilization to provide a stable origin [8,9]. Because the short head of the biceps brachii has a proximal attachment on the coracoid process, scapular stabilizers, such as the serratus anterior and lower trapezius, must provide proximal stabilization for the optimal action of the biceps brachii $[4,10]$. In this study, the SW group showed significant lower scapular protractor strength, which might be the reason for inadequate stabilization and lower elbow flexor strength. However, elbow flexor strength was not included as a predictor variable. Considering that a decision tree selects the predictor variables that maximizes the decrease in impurity [36], in subgroups those who had scapular protractor strength lower than $20 \%$ of body weight, shoulder internal rotation ROM may have been more likely to reduce impurity than elbow flexor strength. Scapular protractor strength and elbow flexor strength are variables that measured muscle strength. On the other hand, shoulder internal rotation ROM is a variable that measured joint flexibility. Therefore, scapular protractor strength and elbow flexor strength represented similar features of participants, but shoulder internal rotation ROM might represent different features of participants from scapular protractor strength and elbow flexor strength. This is a possible reason why there was a significant difference between the two groups, but elbow flexor strength was not included as a predictor variable.

In this study, the classification tree shows acceptable accuracy $(82.35 \%$ in the test data set) and high specificity $(95.65 \%$ in the test data set). However, the classification tree shows low sensitivity (54.55\% in the test data set), which indicates that the model had low performance in correctly identifying subjects with SW. The classification algorithms usually exhibit poor performance while dealing with imbalanced class data $(33$ cases had SW, and 67 cases were without SW in this study), and results are biased toward the majority class [41]. Although various data mining techniques, such as upsampling and downsampling, could be applied to achieve better performance with imbalanced class, we did not use additional techniques to 
measure the data. Moreover, we assigned the scapula with a cutoff value of $1.5 \mathrm{~cm}$ based on the previous study [21]. When compared to a cut off value of $2 \mathrm{~cm}$, the more subjects with less severe SW could be assigned to the SW group with a cut off value of $1.5 \mathrm{~cm}$. Therefore, the boundary value near the cutoff value could make accurate classification difficult. These were possible reasons for low sensitivity of the classification tree.

The current study has several limitations. First, the sample size is small. Because data mining techniques are powerful in terms of uncovering interesting patterns and relationships hidden in a large volume of data, further studies with a large number of subjects are needed. Second, we analyzed only static SW condition. Scapular dyskinesis have two separate entities, static position and dynamic motion. Scapular stabilization during arm motions is important for optimal movements. Therefore, future research is required to investigate the differences between subjects with and without SW in dynamic conditions.

\section{CONCLUSIONS}

The classification tree showed acceptable accuracy (82.35\%) and high specificity (95.65\%) but low sensitivity (54.55\%). Scapular protractor strength, shoulder internal rotation ROM, and whether the scapula is in the dominant or nondominant side were predictors for presence of SW. Based on the predictive model in this study, we suggested that $20 \%$ of body weight in scapular protractor strength is a meaningful cutoff value for presence of SW.

\section{CONFLICTS OF INTEREST}

No potential conflict of interest relevant to this article was reported.

\section{AUTHOR CONTRIBUTIONS}

Conceptualization: GG. Data curation: GG, YW. Formal analysis: GG, YW. Investigation: GG, JK. Methodology: GG, SA, JK, OK. Project administration: GG, OK. Resources: SA, JK, OK. Software: GG. Supervision: OK. Validation: SA, JK, OK. Visualization: GG. Writing - original draft: GG. Writing - review \& editing: OK.

\section{ORCID}

Gyeong-tae Gwak, https://orcid.org/0000-0003-1812-8910

Sun-hee Ahn, https://orcid.org/0000-0001-7660-8598

Jun-hee Kim, https://orcid.org/0000-0002-0498-7207

Young-soo Weon, https://orcid.org/0000-0003-3852-606X

\section{REFERENCES}

1. Kibler WB, Sciascia A. Current concepts: scapular dyskinesis. Br J Sports Med 2010;44(5):300-5.

2. Kibler WB, Sciascia A, Wilkes T. Scapular dyskinesis and its relation to shoulder injury. J Am Acad Orthop Surg 2012;20(6): 364-72.

3. Lukasiewicz AC, McClure P, Michener L, Pratt N, Sennett B. Comparison of 3-dimensional scapular position and orientation between subjects with and without shoulder impingement. J Orthop Sports Phys Ther 1999;29(10):574-83.

4. Kibler WB, Ludewig PM, McClure PW, Michener LA, Bak K, Sciascia AD. Clinical implications of scapular dyskinesis in shoulder injury: the 2013 consensus statement from the 'scapular summit'. Br J Sports Med 2013;47(14):877-85.

5. Hardwick DH, Beebe JA, McDonnell MK, Lang CE. A comparison of serratus anterior muscle activation during a wall slide exercise and other traditional exercises. J Orthop Sports Phys Ther 2006;36(12):903-10.

6. Ha SM, Kwon OY, Cynn HS, Lee WH, Park KN, Kim SH, et al. Comparison of electromyographic activity of the lower trapezius and serratus anterior muscle in different arm-lifting scapular posterior tilt exercises. Phys Ther Sport 2012;13(4):22732.

7. Park KM, Cynn HS, Kwon OY, Yi CH, Yoon TL, Lee JH. Comparison of pectoralis major and serratus anterior muscle activities during different push-up plus exercises in subjects with and without scapular winging. J Strength Cond Res 2014;28(9):2546-51.

8. Kobesova A, Dzvonik J, Kolar P, Sardina A, Andel R. Effects of shoulder girdle dynamic stabilization exercise on hand muscle strength. Isokinet Exerc Sci 2015;23(1):21-32.

9. Schreiber AL, Abramov R, Fried GW, Herbison GJ. Expanding the differential of shoulder pain: Parsonage-Turner syndrome. J Am Osteopath Assoc 2009;109(8):415-22.

10. Muscolino JE. The muscular system manual: the skeletal muscles of the human body. 4th ed. St. Louis: Mosby; 2016;152-4. 
11. Borich MR, Bright JM, Lorello DJ, Cieminski CJ, Buisman T, Ludewig PM. Scapular angular positioning at end range internal rotation in cases of glenohumeral internal rotation deficit. J Orthop Sports Phys Ther 2006;36(12):926-34.

12. Novak CB. Upper extremity work-related musculoskeletal disorders: a treatment perspective. J Orthop Sports Phys Ther 2004;34(10):628-37.

13. Burn MB, McCulloch PC, Lintner DM, Liberman SR, Harris JD. Prevalence of scapular dyskinesis in overhead and nonoverhead athletes: a systematic review. Orthop J Sports Med 2016;4(2):2325967115627608.

14. Cieminski CJ, Kelly SM, Nawrocki TJ, Indrelie AJ, Klaers H, Stelzmiller MR. Comparison of shoulder internal rotation passive range of motion in various positions in nonathletic persons and the establishment of normative values for the sidelying position. J Shoulder Elbow Surg 2016;25(9):1523-31.

15. Macedo LG, Magee DJ. Differences in range of motion between dominant and nondominant sides of upper and lower extremities. J Manipulative Physiol Ther 2008;31 (8):577-82.

16. Seabra P, Van Eck CF, Sá M, Torres J. Are professional handball players at risk for developing a glenohumeral internal rotation deficit in their dominant arm? Phys Sportsmed 2017;45(2):77-81.

17. Torres RR, Gomes JL. Measurement of glenohumeral internal rotation in asymptomatic tennis players and swimmers. Am J Sports Med 2009;37(5):1017-23.

18. Oyama S, Myers JB, Wassinger CA, Daniel Ricci R, Lephart SM. Asymmetric resting scapular posture in healthy overhead athletes. J Athl Train 2008;43(6):565-70.

19. Shiri R, Varonen H, Heliövaara M, Viikari-Juntura E. Hand dominance in upper extremity musculoskeletal disorders. J Rheumatol 2007;34(5):1076-82

20. Hong J, Barnes MJ, Leddon CE, Van Ryssegem G, Alamar B. Reliability of the sitting hand press-up test for identifying and quantifying the level of scapular medial border posterior displacement in overhead athletes. Int J Sports Phys Ther 2011;6(4):306-11.

21. de Oliveira VMA, Beltrão NB, dos Passos MHP, da Silva JP, Pitangui ACR, de Araújo RC. Validity and reliability of an instrument to assess the posterior scapular displacement. Int J Phys Ther Rehab 2015;1:106.

22. Plafcan DM, Turczany PJ, Guenin BA, Kegerreis S, Worrell TW. An objective measurement technique for posterior scapular displacement. J Orthop Sports Phys Ther 1997;25(5):336-41.
23. Weon JH, Kwon OY, Cynn HS, Lee WH, Kim TH, Yi CH. Realtime visual feedback can be used to activate scapular upward rotators in people with scapular winging: an experimental study. J Physiother 201 1;57(2):101-7.

24. Kantardzić M. Data mining: concepts, models, methods and algorithms. New York: Wiley-Interscience; 2002;189-91.

25. Mendelek F, Caby I, Pelayo P, Kheir RB. The application of a classification-tree model for predicting low back pain prevalence among hospital staff. Arch Environ Occup Health 2013;68(3):135-44.

26. Ramezankhani A, Pournik O, Shahrabi J, Khalili D, Azizi F, Hadaegh F. Applying decision tree for identification of a low risk population for type 2 diabetes. Tehran Lipid and Glucose Study. Diabetes Res Clin Pract 2014;105(3):391-8.

27. Krishna OB, Maiti J, Ray PK, Mandal S. Assessment of risk of musculoskeletal disorders among crane operators in a steel plant: a data mining-based analysis. Hum Factors Ergon Manuf Serv Ind 2015;25(5):559-72.

28. Zurada J. Classifying the risk of work related low back disorders due to manual material handling tasks. Expert Syst Appl 2012;39(12):11125-34.

29. Zurada J, Karwowski W, Marras W. Classification of jobs with risk of low back disorders by applying data mining techniques. Occup Ergon 2004;4:291-305.

30. Herland M, Khoshgoftaar TM, Wald R. A review of data mining using big data in health informatics. J Big Data 2014;1(1):2.

31. Du WY, Huang TS, Hsu KC, Lin JJ. Measurement of scapular medial border and inferior angle prominence using a novel scapulometer: a reliability and validity study. Musculoskelet Sci Pract 2017;32:120-6.

32. Jung SH, Hwang UJ, Kim JH, Gwak GT, Kwon OY. Effects of horizontal shoulder abduction and adduction on the activity and strength of the scapular protractors. J Electromyogr Kinesiol 2017;37:155-9.

33. Kim HA, Hwang UJ, Jung SH, Ahn SH, Kim JH, Kwon OY. Comparison of shoulder strength in males with and without myofascial trigger points in the upper trapezius. Clin Biomech (Bristol, Avon) 2017;49:134-8.

34. Jung SH, Kwon OY, Yi CH, Cho SH, Jeon HS, Weon JH, et al. Predictors of dysfunction and health-related quality of life in the flexion pattern subgroup of patients with chronic lower back pain: The STROBE study. Medicine (Baltimore) 2018;97(29):e1 1363.

35. Allaire J. RStudio: integrated development environment for 
R. Paper presented at: The R User Conference, useR! 2011; 2011 Aug 16-18; Coventry, UK: University of Warwick, 2011. p. 14 .

36. Timofeev R. Classification and regression trees (CART) theory and applications. Berlin, Humboldt University, Master's thesis. 2004.

37. Kim JS, Ahn DH, Park DH, Oh JS. Electromyographic activity of the serratus anterior and pectoralis major during isometric scapular protraction at different resistance intensities in subjects with and without a winged scapula. Clin Biomech (Bristol, Avon) 2019;61:199-204.

38. Kim YG, Kang MH, Kim JW, An DH, Oh JS. Shoulder girdle protraction strength and dynamic performance of the upper limb in individuals with scapular winging: A preliminary study.
Isokinet Exerc Sci 2015;23(1):33-40.

39. Magermans DJ, Chadwick EK, Veeger HE, van der Helm FC. Requirements for upper extremity motions during activities of daily living. Clin Biomech (Bristol, Avon) 2005;20(6):5919.

40. Namdari S, Yagnik G, Ebaugh DD, Nagda S, Ramsey ML, Williams GR Jr, et al. Defining functional shoulder range of motion for activities of daily living. J Shoulder Elbow Surg 2012;21(9):1177-83.

41. Wang KJ, Makond B, Wang KM. An improved survivability prognosis of breast cancer by using sampling and feature selection technique to solve imbalanced patient classification data. BMC Med Inform Decis Mak 2013;13:124. 